\title{
A SPECIAL CLASS OF NEAR RINGS
}

\author{
STEVE LIGH
}

(Received 24 November 1972, revised 21 June 1973)

Communicated by G. E. Wall

\section{Introduction}

A near ring is a triple $(R,+, \cdot)$ such that $(R,+)$ is a group, $(R, \cdot)$ is a semigroup, and - is left distributive over + ; i.e. $w(x+z)=w x+w z$ for each $w, x, z$ in $R$. A normal subgroup $K$ of a near ring $R$ is an ideal if (i) $(m+k) n-m n$ is in $K$ for all $m, n$ in $R$ and $k$ in $K$, and (ii) $R K \subseteq K$. In particular, kernels of near ring homomorphisms are ideals. For various other definitions and elementary facts about near rings, see $[5,8]$. For each $x$ in a near ring $R$, let $A(x)$ $=\{y \in R: x y=0\}$. A survey on several recent papers on near rings $[2,3,6,7,8]$ shows that the concept of $A(x)$ being an ideal was the main technique. The purpose of this note is to initiate a study of near rings having the property that each $A(x)$ is an ideal.

A duo ring is a ring in which every one-sided ideal is two-sided. Clearly every duo ring has the property that each $A(x)$ is an ideal. However, the converse is not true, as demonstrated by the non-commutative ring defined on the Klein group $K=\{0, a, b, c\}$ with the following multiplication: $x 0=0, x a=x, x b=0$ $x c=x$ for each $x$ in $K$. Duo rings have been studied in [11] while application of duo rings to commutativity theorems was given in [1]. This is another motivation for the study of near rings with the property that each $A(x)$ is an ideal.

\section{Main results}

In this section we restrict our attention to near rings with d.c.c. on $R$-subgroups. To facilitate the discussion, we begin with some definitions.

DEFINITION 2.1. The near ring $R$ is said to have property $p$ if $A(x)$ is an ideal of $R$ for each $x$ in $R$.

DEFINITION 2.2. An ideal I of a near ring is called trivial if $x y=0$ for each $x, y$ in $I$.

LEMMA 2.3. Let $R$ be a near ring with property $p$. If $e^{2}=e$, then exy $=$ exey for all $x, y$ in $R$ and the set $K=\{e r-r: r \in R\}$ is an ideal of $R$. 
ProOF. If $e$ is a left identity, then conclustion is trivial. If $e$ is not a left identity, then $A(e)$ is an ideal. Thus $\operatorname{ex}(e y-y)=0$ implies that $e x y=e x e y$. Now define the map $F: R \rightarrow e R$ by $r F=e r$. Then $F$ is an onto near ring homomorphism and the kernel of $F$ is precisely $K$.

Before we proceed further, a definiton is needed.

DEFINITION 2.4. Let $R$ be a near ring. If $x R=R$ for each $x$ in $R$ or $0 R=0$ and $x R=R$ for each $x \neq 0$ in $R$, then $R$ is said to be of type I. If $x R=R$ for some $x$ in $R$ and $y R=0$ for some $y \neq 0$ in $R$, then $R$ is said to be of type $I I$.

The following theorem, though quite technical in nature, has many interesting corollaries.

THEOREM 2.5. Let $R$ be a near ring with d.c.c. on $R$-subgroups and having property $p$. If each minimal ideal of $R$ is nontrivial, then $R$ is a direct sum of ideals each of which is of either type I or type II.

Proof. If $R$ is simple, then $R$ has no nonzero divisors of zero and for each $x$ in $R$, there is an integer $n$ such that $x^{n} R=x^{n+1} R$. Let $y$ be in $R$. Then there is a $t$ in $R$ such that $x^{n} y=x^{n+1} t$. Hence $y=x t$ and this implies that $x R=R$. Thus $R$ is of type $I$.

If $R$ is not simple, let $J$ be a minimal ideal. Since $J$ is nontrivial, there are elements $x, y$ in $J$ such that $x y \neq 0$. Since $A(x)$ is an ideal of $R$, it follows that $A(x) \cap J=0$. Since $x J$ is an $R$-subgroup, we see that $x J=J$. Thus there is an element $e$ in $J$ such that $e^{2}=e$. Now $e J \subseteq e R \subseteq J$ implies that $e R=J$. By Lemma 2.3, it is easy to show that $R=J \oplus K$, where $K=\{e r-r: r \in R\}$. Let $y$ be in $J$. Then $A(y) \cap J=0$ or $A(y) \cap J=J$. At any rate $J$ is either of type I or II. Now since $K$ is an ideal of $R$, and any ideal of $K$ is an ideal of $R$, we see that $K$ inherits the properties of $R$. Thus our decomposition continues and the conclusion follows.

We begin with a corollary which is immediate.

COROLlARY 2.6. Let $R$ be a near ring with d.c.c. on $R$-subgroups and having property $p$. Then $R$ is a direct sum of near rings $R_{i}$ where $R_{i}$ is either of type I or II or every minimal ideal of $R_{i}$ is trivial.

REMARK 2.7. From the introduction we see that there are rings which have property $p$ and every minimal ideal is trivial, furthermore, they are indecomposable.

COROLlary 2.8. Let $R$ be a near ring with no nonzero nilpotent elements and $0 x=0$ for each $x$ in $R$. If $R$ has d.c.c. on $R$-subgroups, then $R$ is a direct sum of ideals $R_{i}$ where each $R_{i}$ is one of the followings: (1) $R_{i}$ is a near field, (2) $R_{i}$ is a near integral domain and (3) each element is a left identity in $R_{i}$.

PROOF. It is easily shown that $R$ has property $p$ and every minimal ideal of 
$R$ is nontrivial. Thus by Theorem 2.5 , each $R_{i}$ is of type $I$, that is, $x R_{i}=R_{i}$ for all $x \neq 0$ in $R_{i}$. If $R_{i}$ has a nonzero right distributive element, by [4, Theorem 2.3] $R_{i}$ is a near field. If $R_{i}$ has a nontrivial multiplication, then $R_{i}$ is a near integral domain [9]. If $R_{i}$ is neither one of the above, then every element is a left identity.

Since every element of a ring is right distributive, the following result is immediate.

COROllaRY 2.9. Let $R$ be a ring with no nonzero nilpotent elements. If d.c.c. for right ideals holds in $R$, then $R$ is a direct sum of division rings. In particular, if $R$ is finite, then $R$ is commutative and is a direct sum of ideals.

COROLlaRY 2.10. Let $R$ be a ring with d.c.c. on right ideals. Then the following are equivalent:

(1) $R$ has no nonzero nilpotent elements

(2) $R$ is a direct sum of division rings

(3) $R$ has property $p$ and every minimal ideal is nontrivial.

Proof. In view of Corollary 2.9, we need to show only (3) implies (2). Suppose $R$ satisfies (3). If $R$ is simple, then $R$ has no nonzero zero divisors. Thus for each $x \neq 0$ in $R, x R=R$ implies that $R$ is a division ring. If $R$ is not simple then by Theorem $2.5, R$ is a direct sum of ideals $J_{n}$ each of which is of type $I$ or type II. We wish to show that $J_{n}$ are of type I. Suppose $J$ is a minimal ideal and there is a $y \neq 0$ in $J$ such that $y J=0$. Now consider $L(J)=\{j \in J: j x=0$ for all $x \in J\}$. It can be verified easily that $L(J)$ is an ideal of $R$. Thus $L(J) \cap J$ $=J$ or $L(J) \cap J=0$. Since $y J=0$ it follows that $L(J) \cap J=J$. But this implies that $J$ is trivial, a contradiction. Hence $x J=J$ for each $x \neq 0$ in $J$ and thus $J$ is a division ring.

It was shown in [9] that the additive group of a finite near integral domain is nilpotent. The following is immediate from Corollary 2.8 .

COROLlARY 2.11. Let $R$ be a finite near ring with no nonzero nilpotent elements and $0 x=0$ for each $x$ in $R$. If every homomorphic image of $R$ has a nontrivial multiplication (i.e. not every element is a left identity), then $R$ is a direct sum of near integral domains and hence $(R,+)$ is nilpotent. In particular, if $R$ has an identity, then $R$ is a direct sum of near fields.

There are many more interesting results concerning near rings with identities and d.g. near rings with d.c.c. and finite near rings which can be obtained from Theorem 2.5 easily, but it is not necessary to state them all explicitely here. We conclude this section with the following result which follows easily from Theorem 2.5 .

COROLLARY 2.12. Let $R$ be a regular near ring with d.c.c. on $R$-subgroups and idempotents are central. Then $R$ is a direct sum of near fields. 


\section{Remarks}

Definition 3.1. A near ring $R$ is said to have property $q$ if every homomorphic image $R^{\prime}$ of $R$ satisfies: $A(x)$ is an ideal for each $x$ in $R^{\prime}$.

We now state a structure theorem for near rings with property $q$.

THEOREM 3.2. A near ring $R$ with property $q$ is isomorpic to a subdirect sum of near rings $R_{i}$ where each $R_{i}$ is one of the following types:
A. each element is a left identity,
B. $0 x=0$ for all $x$ in $R_{i}$ and $R_{i}$ has no nonzero divisors of zero,
C. the intersection of all proper ideals has no nonzero idempotents,
D. if there is $e^{2}=e$ in the intersection I of all proper ideals, then $I=A(0)$.

It can be shown now many of the results in $[2,3,5,6,7,8,10]$ are corollaries of the above theorem.

\section{References}

[1] H. E. Bell, 'Duo rings: some applications to commutativity theorems', Canad. Math. Bull. 11 (1968), 375-380.

[2] H. E. Bell, 'Near rings in which each element is a power of itself', Bull. Austral. Math. Soc. 2 (1970), 363-368.

[3] H. E. Bell, 'Certain near rings are rings', J. London Math. Soc. (2), 4 (1971), 264-270.

[4] S. Ligh, 'On division near rings', Canad. J. Math. 21 (1969), 1366-1371.

[5] S. Ligh, 'On boolean near rings', Bull. Austral. Math. Soc. 1 (1969), 375-279.

[6] S. Ligh, 'On regular near rings', Math. Japan. 15 (1970), 7-13.

[7] S. Ligh, 'On the commutativity of near rings, III.', Bull. Austral. Math. Soc. 6(1972), 459-464.

[8] S. Ligh, 'The structure of a special class of near rings', J. Austral. Math. Soc. 13 (1972), 141-146.

[9] S. Ligh, 'On the additive group of finite near integral domains and simple d.g. near rings', Monatsh. Math. 76 (1972), 317-322.

[10] G. Szeto, 'On a class of near rings', J. Austral. Math. Soc. 14 (1972), 17-19.

[11] G. Thierrin, 'On duo rings', Canad. Math. Bull. 3 (1960), 167-172.

University of Southwestern Lousiana

Lafayette, Lousiana 70501

U. S. A. 